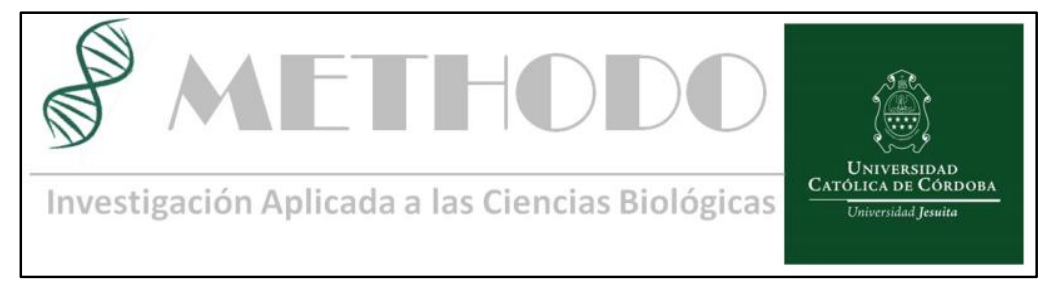

\title{
Uso del cannabis en 16 pacientes con epilepsia refractaria
}

\section{Use of cannabis in 16 patients with refractory epilepsy.}

\author{
Vaudagna $\mathrm{M} \mathrm{P}^{1-2}$, Vergara $\mathrm{G}^{1-2}$, Martínez $\mathrm{A}^{1-2}$, Paviolo M1-2, Miculan $\mathrm{J}^{1-2}$, Linzoain $\mathrm{J}^{1}$, Sfaello Z, \\ Sfaello $\mathrm{I}^{1-2}$
}

\section{Introducción:}

En la actualidad, el 30 a $40 \%$ de los pacientes epilépticos son refractarios a los fármacos anticonvulsivantes. En relación a esto, en los últimos años el uso de

marihuana medicinal se ha convertido en un tema controversial tanto en la comunidad médica como en la población general.

Se ha descripto que el sistema endocannabinoide del cerebro proporciona protección contra la actividad convulsiva, desempeñando un papel importante en la regulación del sistema nervioso central y equilibrando la interacción sináptica excitadora e inhibidora para una función cerebral normal. El mismo está compuesto principalmente de dos receptores cannabinoides: CB1R (Receptor cannabinoide tipo 1) y CB2R (Receptor Cannabinoide tipo 2). Los receptores CB1R se expresan predominantemente en sistema nervioso central, y el receptor CB2R se localiza principalmente en el sistema inmune humano $\mathrm{y}$ hematopoyético. ${ }^{1}$

La marihuana tiene dos componentes principales que influyen en el sistema endocannabinoide: delta-9-tetrahidrocannabinol (THC) y el cannabidiol (CBD). El THC a través del CB1R ejerce su función psicoactiva. Sin embargo, el CBD presenta baja afinidad y es antagonista de CB1R y CB2R. Algunos estudios en animales muestran que los antagonistas de CB1R pueden reducir el umbral de las convulsiones. El CBD actúa a través de múltiples mecanismos. Estos incluyen efectos sobre los canales Potasio, Calcio, y funciones antagónicas en el receptor acoplado a proteína $\mathrm{G}$, mediante el cual puede disminuir la liberación pre sináptica de glutamato, excitador del sistema nervioso central. Con todo, los mecanismos exactos subyacentes a los efectos antiepilépticos del CBD no están bien definidos. ${ }^{2}$ En estudios iniciales realizados con utilización del extracto de aceite de cáñamo (con concentraciones de $\mathrm{THC}<0,3 \%$ ), en pacientes con Síndrome de Dravet y Epilepsia tipo Lennox Gastaut, se reportó una franca mejoría en la frecuencia de las crisis y en la calidad de conexión con el entorno del paciente. Asimismo, se objetivó alrededor de un $10 \%$ de efecto placebo debido a una escaza confiabilidad en el diseño de dichos estudios, mayormente basados en encuestas. Actualmente los últimos estudios son realizados con CBD sintetizado, sin contener THC y a dosis mayores, con resultados prometedores. ${ }^{3}$

\section{Objetivos:}

Establecer la respuesta del tratamiento coadyuvante mediante la utilización de Cannabis, Aceite de Charlotte, en pacientes pediátricos con diagnóstico de epilepsia refractaria. Evaluar efectos adversos y positivos no convulsivos en los pacientes estudiados.

\section{Materiales y métodos:}

Se realizó un trabajo retrospectivo, descriptivo, desde agosto del 2016 hasta agosto de 2017.

Se incluyeron pacientes de inicio de epilepsia en edad pediátrica, con un rango etario desde el año hasta los 25 años, con diagnóstico de epilepsia refractaria de más de un año de evolución, de diversas etiologías. Se definió refractariedad como la persistencia de crisis pese al uso de dos o más fármacos anticonvulsivantes adecuados al tipo de epilepsia. 
Se indicó como tratamiento coadyuvante cannabidiol gotas, Every day Advanced 5000,

(único aceite de cáñamo aprobado por la ANMAT para la importación). La dosis utilizada fue de 0,25 $\mathrm{mg} / \mathrm{lb} /$ día hasta $1 \mathrm{mg} / \mathrm{lb} /$ día. La dosis se planteó según la indicación dada por el laboratorio Charlotte Web, iniciando con $0.25 \mathrm{mg} / \mathrm{lb} / \mathrm{dí}$, titulando la misma cada 15 días sino se observaba mejoría clínica en el paciente.

Se evaluaron las frecuencias de las crisis y los electroencefalogramas (EEG) previos y

posteriores al tratamiento con CBD. Se consideró mejoría de las crisis como la disminución en la frecuencia de las mismas en un 50\% o más.

El análisis estadístico se realizó con el programa R-Medic para evaluar la frecuencia de crisis, prueba de probabilidad ( $\mathrm{p}<0.5$ ). Para el análisis del EEG control, se utilizó el test de proporciones.

Análisis estadístico: Se realizó estadística descriptiva. Para las variables se utilizó prueba de Chi cuadrado. Se consideró significación estadística $\mathrm{p}<0,05$.

\section{Resultados:}

Del total de la muestra $(n=16)$ en el periodo establecido, la media de tratamiento fue de 5.4 meses, con un rango de 2 a 12 meses.

Se observó en tres pacientes (19\%) una disminución de igual o mayor al $50 \%$ en la frecuencia de las crisis (p1).

Nueve pacientes asistieron a control con registro electroencefalográfico, de los cuales dos pacientes presentaron cambios positivos en los trazados. Al relacionar estos cambios electroencefalográficos con la frecuencia de la crisis, se observó una mejoría estadísticamente significativa $(\mathrm{p}<0.01)$.

Además, en cinco pacientes $(31 \%)$ hubo otros efectos positivos, siendo el más frecuente la mejoría en la conexión con el entorno.

El efecto adverso más frecuente fue irritabilidad que se observó en 6 pacientes $(37,5 \%)$.

Cinco pacientes $(31 \%)$ decidieron abandonar el tratamiento; 4 en menos de 4 meses de seguimiento, debido a la falta de respuesta en la frecuencia de las crisis.

\section{Discusión:}

La falta de ensayos de calidad publicados en la literatura, hace que persista la incertidumbre sobre el uso terapéutico de productos cannabinoides en la población pediátrica. En su mayoría corresponden a estudios de datos retrospectivos de corto seguimiento, basados en encuestas a padres sobre de las características de las convulsiones del niño y condiciones de comportamiento.

Resultados similares a los nuestros, arrojo el ensayo publicado por Chapman y cols. ${ }^{4}$ uno de los primeros trabajos presentado con canabidiol gotas Every day Avdanced 5000, en el cual, de un total de 119 pacientes, en $24(20 \%)$ hubo una disminución de menos del $50 \%$ de crisis epilépticas. Esto se observó predominantemente en epilepsia tipo lennox-gastaut, a diferencia de nuestros pacientes, los cuales padecían epilepsia refractaria secundaria a causa estructural.

En el presente estudio, en los pacientes con diagnóstico de Síndrome de Dravet, no se identificaron resultados beneficiosos con el tratamiento coadyuvante; a diferencia de Cross y cols5 en el que se informó una mejoría en la reducción de las crisis en $29(47.5 \%)$ de 61 pacientes tratados con CBD sintetizado, con un rango de dosis de $10-20 \mathrm{mg} / \mathrm{kg} /$ día. Se desconoce si dicha mejoría se debe al aumento en las dosis usadas, o al tipo de fármaco utilizado.

Con respecto a los efectos positivos no convulsivos, no se observaron diferencias con los estudios previamente mencionados. ${ }^{6-7}$

En nuestro estudio, el principal efecto adverso fue la irritabilidad a diferencia de los trabajos de referencia citados, en los que el efecto adverso más frecuente fue la somnolencia y los síntomas gastrointestinales, los cuales a su vez llevaron a suspender la medicación. La suspensión de la medicación en nuestro estudio fue por falta de respuesta en el control de crisis.

\section{Conclusión:}

No se determinarón resultados significativos del cannabidiol en el manejo de las crisis refractarias ni en los trazados de EEG. Sí se observó beneficio terapéutico en un grupo de pacientes con epilepsia debido a causa estructural.

Sería importante realizar investigaciones de ensayos ciegos, prospectivos, aleatorizados $\mathrm{y}$ controlados, con seguimiento a largo plazo para obtener respuestas de mayor contundencia a los objetivos planteados, evaluando la concentración de CBD y THC. utilizada, la dosis, las interacciones medicamentosas con drogas antiepilépticas y repercusión en trazados EEG.

\section{Introduction:}

Currently, 30 to $40 \%$ of epilepsy patients are refractory to anti - convulsant drugs.

In recent years, the use of medical marijuana has become a controversial issue not only within the medical community but also in the general population.

Revista Methodo: Investigación Aplicada a las Ciencias Biológicas. Universidad Católica de Córdoba.

Jacinto Ríos 571 Bo Gral. Paz. X5004FXS. Córdoba. Argentina. Tel.: (54) 3514517299 / Correo:

methodo@ucc.edu.ar / Web: methodo.ucc.edu.ar | ARTICULO ORIGINAL Methodo 2018;3(2):29-31 
Cannabidiol it would be responsible for the anticonvulsant effect through modulation of the endocannabinoid system.

\section{Objective:}

To evaluate the efficacy of adjuvant treatment cannabis in patients with refractory epilepsy.

\section{Materials and methods:}

A descriptive retrospective study was performed from August 2016 to August 2017. Patients with a diagnosis of refractory epilepsy of various etiologies, to which was indicated as adjunctive therapy cannabidiol drops Everyday Advanced 5000 at a dose of $0.25 \mathrm{mg} / \mathrm{lb} / \mathrm{day}$.

\section{Statistical analysis:}

Descriptive statistics was performed. For variables a chi-square test was used. Statistical significance $\mathrm{p}<0.05$ was considered.

\section{Results:}

The total sample $(\mathrm{n}=16), 19 \%$ (3 patients) had a reduction equal to or greater than $50 \%$ in the seizure frequency $(p=1)$.

Nine patients had electroencephalogram control, of which two patients showed improvements in drawn. By linking patients with EEG control and seizure frequency during treatment, a correlation statistically signific observed, with $\mathrm{p}<0.01$.

The $31 \%$ showed other positive effects reported by the family, the most common, improvements in connection with the environment. In 6 patients $(37.5 \%)$ the adverse reaction was irritability.

Five patients $(31 \%)$ decidie rum discontinue treatment in less than 4 months, seeing no significant changes in the frequency of crisis.

\section{Conclusion:}

In this study was not significant efficacy of cannabidiol, on crisis management. Given these results, it is important multicentric prospective studies.

\section{Bibliografía:}

1.Drs. Kolikonda and Sagi, y cools.; "Medical Marijuana for Epilepsy?"; Innov Clin Neurosci. 2016 Mar-Apr; 13(3-4): 23-26; Chung Mo Koo1 and Hoon-Chul Kang; "Could Cannabidiol be a Treatment Option for Intractable Childhood and
Adolescent Epilepsy?"; J Epilepsy Res. 2017 Jun; 7(1): 16-20; DOI:10.14581/jer.17003

2. Chung Mo Koo1 and Hoon-Chul Kang;" Could Cannabidiol be a Treatment Option for Intractable Childhood and Adolescent Epilepsy?"; J Epilepsy Res. 2017 Jun; 7(1): 16-20; Published online 2017 Jun 30. doi: 10.14581/jer.17003

3. Christopher T. Campbell, "Cannabinoids in Pediatrics"; J Pediatr Pharmacol Ther 2017;22(3):176-185; DOI: 10.583/1551-677622.3.176

4. Lauren Treat, Kevin E. Chapman y cools; "Duration of use of oral cannabis extract in a cohort of pediatric epilepsy patients"; Wiley Online Library; DOI:10.1111/epi.13617

5. Orrin Devinsky, MD, J. Helen Cross, y cools. "Trial of Cannabidiol for Drug-Resistant Seizures in the Dravet Syndrome"; N Engl J Med 2017; 376:2011-2020; DOI: 10.1056/NEJMoa1611618

6. Dr Prof. Orrin Devinsky, Eric Marsh, y cools.; "Cannabidiol in patients with treatment-resistant epilepsy: an open-label interventional trial"; The Lancet Neurology; Volume 15, No. 3, p270-278, March 2016; DOI: https://doi.org/10.1016/S14744422(15)00379-8

7. O'Connell BK y cools; "Cannabinoids in treatment-resistant epilepsy: A review"; Epilepsyehav (2016) http://dx.doi.org/10.1016/j.yebeh.2016.11.012

\section{Palabras claves:}

Cannabis, Encefalopatía Epiléptica.

\section{Keywords:}

Cannabis, Epileptic Encephalopathy.

1Servicio de Neurología Infantil, Instituto de Neurología Infanto Juvenil, CETES. Córdoba, Argentina.

2Servicio de Neurología Infantil Clínica Universitaria Reina Fabiola. Córdoba, Argentina

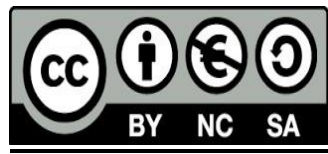

